\title{
XML Bound
}

National Cancer Institute

\section{Source}

National Cancer Institute. XML Bound. NCI Thesaurus. Code C80696.

Indicates that information in an XML document is represented as an object in computer memory. 